\title{
Effect of anti-diabetic drugs in dialysis patients with diabetes: a nationwide retrospective cohort study
}

\author{
Shih-Hsiang Ou ${ }^{1,2,3}$, Hsin-Yu Chen ${ }^{1}$, Nai-Wen Fang ${ }^{4}$, Chun-Hao Yin ${ }^{5}$, Chien-Liang Chen ${ }^{1,2}$ and \\ Jin-Shuen Chen ${ }^{1,5,6^{*}}$
}

\begin{abstract}
Background: Type 2 diabetes mellitus is common in patients undergoing dialysis. However, the association between anti-diabetic drug use and survival outcomes is rarely discussed. We aimed to investigate whether continued antidiabetic medication use affects the survival of diabetic dialysis patients and whether different hypoglycemic drug use influences prognosis.

Methods: Using a nationwide database, we enrolled patients with incident end-stage renal disease under maintenance dialysis during 2011-2015 into the pre-existing diabetes dialysis (PDD), incident diabetes after dialysis (IDD), and non-diabetic dialysis (NDD) groups. The PDD group was further subclassified into patients who continued (PDD$\mathrm{M})$ and discontinued (PDD-NM) anti-diabetic drug use after dialysis.
\end{abstract}

Results: A total of 5249 dialysis patients were examined. The PDD-NM group displayed a significantly higher mortality rate than the IDD, PDD-M, and NDD groups (log-rank test $P<0.001$ ). The PDD-M group had a significantly lower risk of death, regardless of insulin $(P<0.001)$ or oral hypoglycemic agent $(O H A)(P<0.001)$ use. Initial insulin administration or OHA had no statistically significant effect on overall mortality in the IDD group. But OHA use had better survival trends than insulin administration for the older $(P=0.02)$ and male subgroups $(P=0.05)$.

Conclusions: For dialysis patients with diabetes, continuous administration of anti-diabetic drugs after dialysis and choice of medication may affect outcomes.

Keywords: End-stage renal disease, Dialysis, Type 2 diabetes mellitus, Anti-diabetic drugs

\section{Background}

The worldwide incidence and prevalence of end-stage renal disease (ESRD) have been increasing annually. Type 2 diabetes mellitus (T2DM) is the leading cause of ESRD in most developed countries and one of the common comorbidities in dialysis patients $[1,2]$. Most patients are diagnosed with T2DM before the initiation of dialysis; however, some develop new-onset diabetes afterward.

\footnotetext{
*Correspondence: dgschen@vghks.gov.tw

${ }^{1}$ Division of Nephrology, Department of Internal Medicine, Kaohsiung Veterans General Hospital, Kaohsiung, Taiwan

Full list of author information is available at the end of the article
}

This situation is becoming more common as the survival time of ESRD patients is increasing. Some epidemiologic studies reported an association between pre-existing diabetes in dialysis patients and a poor prognosis [3, 4]. But discussions on clinical outcomes between patients with new-onset diabetes after entering dialysis and other dialysis groups are scarce.

Good glycemic control in diabetic patients is proven to be associated with fewer complications, decreased incidence of cardiovascular disease and mortality, regardless of renal function $[5,6]$. In some cases, diabetic patients may experience spontaneous resolution of hyperglycemia 
with near-normal hemoglobin A1C (HbA1C) when their renal function declines gradually [7]. Such patients are also prone to hypoglycemia after using some glucoselowering drugs, which may hinder continued medication use when renal failure [8]. The biological significance and clinical implications of this phenomenon are unclear [9]. Currently, few studies have explored the clinical difference and prognosis between patients with continued anti-diabetic medication administration after initiation of dialysis and those without.

According to the Kidney Disease Outcomes Quality Initiative (KDOQI) guideline, only several oral hypoglycemic agents (OHA) kinds can be administered in dialysis patients, including sulfonylurea (SU, such as glipizide and gliclazide), meglitinide (repaglinide), thiazolidinediones (TZD), and dipeptidyl-peptidase IV (DPP-4) inhibitors [10]. Most studies focused only on the impact of a certain anti-diabetic drug in this population; whether the choice of different kinds of OHA or insulin will affect the mortality of dialysis patients in different periods remains unknown.

The purpose of this nationwide retrospective study was to use a large dataset from Taiwan's National Health Insurance Research Database (NHIRD) to explore whether continuing anti-diabetic medication administration after initiation of dialysis affects the survival of dialysis patients with pre-existing diabetes, and whether administering different hypoglycemic drugs influences prognoses. Additionally, we aimed to investigate the risk factors associated with the mortality of dialysis patients with pre-existing and new-onset diabetes.

\section{Methods}

\section{Data collection}

This observational cohort study used the "Longitudinal Health Insurance Database 2010 (LHID2010)", established from NHIRD by the Health and Welfare Data Science Center (HWDC) in Taiwan. The Taiwan National Health Insurance (NHI) Program was implemented in 1995, offering comprehensive medical care coverage to more than $99 \%$ of the country's population of 23 million inhabitants. The LHID2010 contains all the original claims data of 2 million beneficiaries randomly sampled from individuals covered by the NHI Program between January 1 and December 31, 2010. The LHID2010 contains integral information, including demographic data, diagnostic codes, registration files, detailed prescriptions, dates of clinical visits, dates of admission and discharge, and medical expenditures for the enrollees of two million beneficiaries. No statistically significant difference was found in the distributions of age and sex between the cohort in the LHID2010 and the Taiwan NHI enrollees. Several scientific research articles have been published and confirmed the validation of the NHIRD database in the T2DM with ESRD population [11, 12]. The disease and diagnostic codes recorded in the registry of clinical visits and hospital care were based on the International Classification of Diseases, Ninth and Tenth Revision, Clinical Modifications (ICD-9-CM and ICD-10-CM). To ensure patient confidentiality, only data with encoded identification numbers were released. The HWDC approved the application after reviewing all the required medical documents. This study was also approved by the Institutional Review Board of the Kaohsiung Veterans General Hospital (IRB No.: KSVGH18-CT10-07), who supervised the study in accordance with the tenets of the Declaration of Helsinki (1975) and its later amendment (2013). The requirement of informed consent was waived.

\section{Study design}

This observational study enrolled patients who began incident maintenance dialysis (hemodialysis or peritoneal dialysis) between January 1, 2011 and December 31, 2015 , through outpatient claims. ESRD patients on maintenance dialysis were defined as having undergone dialysis for more than 90 days. The exclusion criteria included patients who were younger than 20 years; diagnosed with type 1 diabetes mellitus; had undergone maintenance dialysis before tracking; and survived less than 90 days after confirmed maintenance dialysis. The remaining patients were followed up from the day of their first dialysis treatment until their death, dialysis withdrawal, or the end of follow-up (December 31, 2016).

The study participants were classified into patients with T2DM diagnosed before the initiation of dialysis (pre-existing diabetes dialysis group; PDD) and without (non-pre-existing diabetes dialysis group; NPDD). T2DM was defined as having had at least one admission code, or three or more outpatient codes for T2DM (ICD-9: 250.X; ICD-10: E11.X), as well as having received antidiabetic drugs for at least 90 days in the last year before initiation of dialysis. In the PDD group, we separated participants into two groups: continued anti-diabetic drug use after dialysis (pre-existing diabetes dialysis patients continuing medication; PDD-M) and discontinued use (pre-existing diabetes dialysis patients not continuing medication; PDD-NM). The definition of continued medication use was having a record of anti-diabetic drug use for at least 90 days in one year after initiation of dialysis. In the NPDD group, we also identified the patients with new-onset diabetes (incident diabetes after dialysis; IDD) during the follow-up period. New-onset diabetes after entering dialysis was defined as T2DM diagnosed at least three months after dialysis initiation, confirmed by diagnostic codes, and anti-diabetic drug administration history for at least 90 days in one year as previously defined. 
The remaining patients did not develop diabetes between dialysis initiation and the end of follow-up (non-diabetes dialysis; NDD). The endpoint was all-cause mortality.

Confounding factors such as sex, age, comorbidities, residence, insured premium, and dialysis modality were adjusted accordingly. Individuals with comorbidities were confirmed from their history of comorbidities as diagnosed by ICD coding in at least one admission code, or three or more outpatient codes, before the initiation of dialysis. ICD codes are provided in Additional file 1: Table S1. We collected the number of hospitalizations during the 12 months leading up to dialysis initiation as the indicator of vulnerability. This study also evaluated whether anti-diabetic drug choice affects mortality. We defined the first time of anti-diabetic drug use more than 90 days as the specific drug group and then subgrouped the patients into the: Insulin, SU, Meglitinide, TZD, and DPP-4 inhibitors groups. The use of angiotensin-converting enzyme inhibitors (ACEI), angiotensin II receptor blockers (ARB), and lipid-lowering drugs was also considered as a covariate factor. The details of the Anatomical Therapeutic Chemical (ATC) code of different drugs are provided in Additional file 1: Table S2.

\section{Data analysis}

The SAS software (SAS System for Windows, version 9.2; SAS Institute, Cary, NC) and SPSS statistical software 22.0.0 (IBM Corp., Armonk, NY, USA) were used to perform statistical analyses in this study. Descriptive statistics were used to analyze the baseline demographic data and the distribution of each variable among the study population. Continuous variables were expressed as mean $\pm \mathrm{SD}$. Multiple comparisons among the study groups were performed using one-way analysis of variance followed by Bonferroni's post hoc test. Categorical variables were described as proportions and compared by $x^{2}$ analysis with Fisher's exact correction. Overall survival rates were calculated using the Kaplan-Meier method, and the differences in survival was determined by the log-rank test. The stepwise Cox regression method was used for the overall analyses of death in the multivariate model. The entry probability was 0.1 and the removal probability was 0.05 . Statistical significance was defined as $\mathrm{P}<0.05$. The hazard ratios (HR) and their $95 \%$ confidence intervals (CIs) from the Cox regression analyses were used as estimates of relative risk.

\section{Results}

\section{Clinical characteristics and survival rates of dialysis} patients during 2011-2015

We identified a total of 5249 patients on incident maintenance dialysis between 2011 and 2015 from the LHID2010 two million people cohort after evaluating the inclusion and exclusion criteria (Fig. 1). Of these, $2956(56.3 \%)$ patients were classified into the PDD group and $2293(43.7 \%)$ into the NPDD group. There were 2601 (88\%) patients in the PDD-M group and 355 (12\%) patients in the PDD-NM group. In the NPDD group, there were $335(14.6 \%)$ patients in the IDD group and 1958 (85.4\%) patients in the NDD group.

Table 1 presents the distribution of demographic characteristics, comorbidities, and mortality outcome in each group: patients in the PDD-NM group were older than the patients in the other groups $(\mathrm{P}=0.002)$; the NDD group had a higher percentage of patients undergoing peritoneal dialysis $(\mathrm{P}<0.001)$; and patients in the PDD group, regardless of PDD-M or PDD-NM, had a higher hospitalization rate more than once in the year before initiation of dialysis, than the patients in the NPDD group $(\mathrm{P}<0.001)$. In addition, the proportion of comorbidities, including hypertension, hyperlipidemia, congestive heart failure, and cerebrovascular accidents was higher in the PDD group than in the NPDD group $(\mathrm{P}<0.001)$. Patients in the PDD-NM group had a lower proportion of ACEI/ $\mathrm{ARB}$ and lipid-lowering drug usage than other groups $(\mathrm{P}<0.001)$.

We conducted a stepwise regression analysis to evaluate the risk factors for mortality in all dialysis patients (Table 2). After multivariable adjustment, the following factors were found to be significant independent predictors of increased mortality: age $>60$ years $(\mathrm{P}<0.001)$; diagnosis of diabetes, pre-existing or newonset $(\mathrm{P}<0.001)$; hospitalization more than once during the 12 months leading up to dialysis initiation $(\mathrm{P}<0.001)$; congestive heart failure $(\mathrm{P}=0.012)$; cerebrovascular accidents $(\mathrm{P}=0.001)$; chronic obstructive pulmonary disease $(\mathrm{P}<0.001)$; chronic liver disease $(\mathrm{P}=0.002)$; and malignancy $(\mathrm{P}<0.001)$. Factors associated with significantly decreased mortality included hypertension $(\mathrm{P}<0.001)$; ACEI or ARB use $(\mathrm{P}<0.001)$; and lipid-lowering drug use $(\mathrm{P}<0.001)$.

A total of 1596 patients died during the follow-up period, and the Kaplan-Meier survival curve of the four groups is shown in Fig. 2. During the post hoc analysis, the PDD-NM group displayed a significantly higher mortality rate than the IDD, PDD-M, and NDD groups (logrank test $\mathrm{P}<0.001$ ).

\section{Comparisons in PDD patients during 2011-2015}

In Table 3, we also used a stepwise regression model to determine the risk factors of mortality in PDD patients. After multivariable adjustment, factors that were significant independent predictors for increased mortality in the PDD group included: age older than 60 years $(\mathrm{P}<0.001)$, discontinued anti-diabetic drug use $(\mathrm{P}<0.001)$, hospitalization more than once during the 


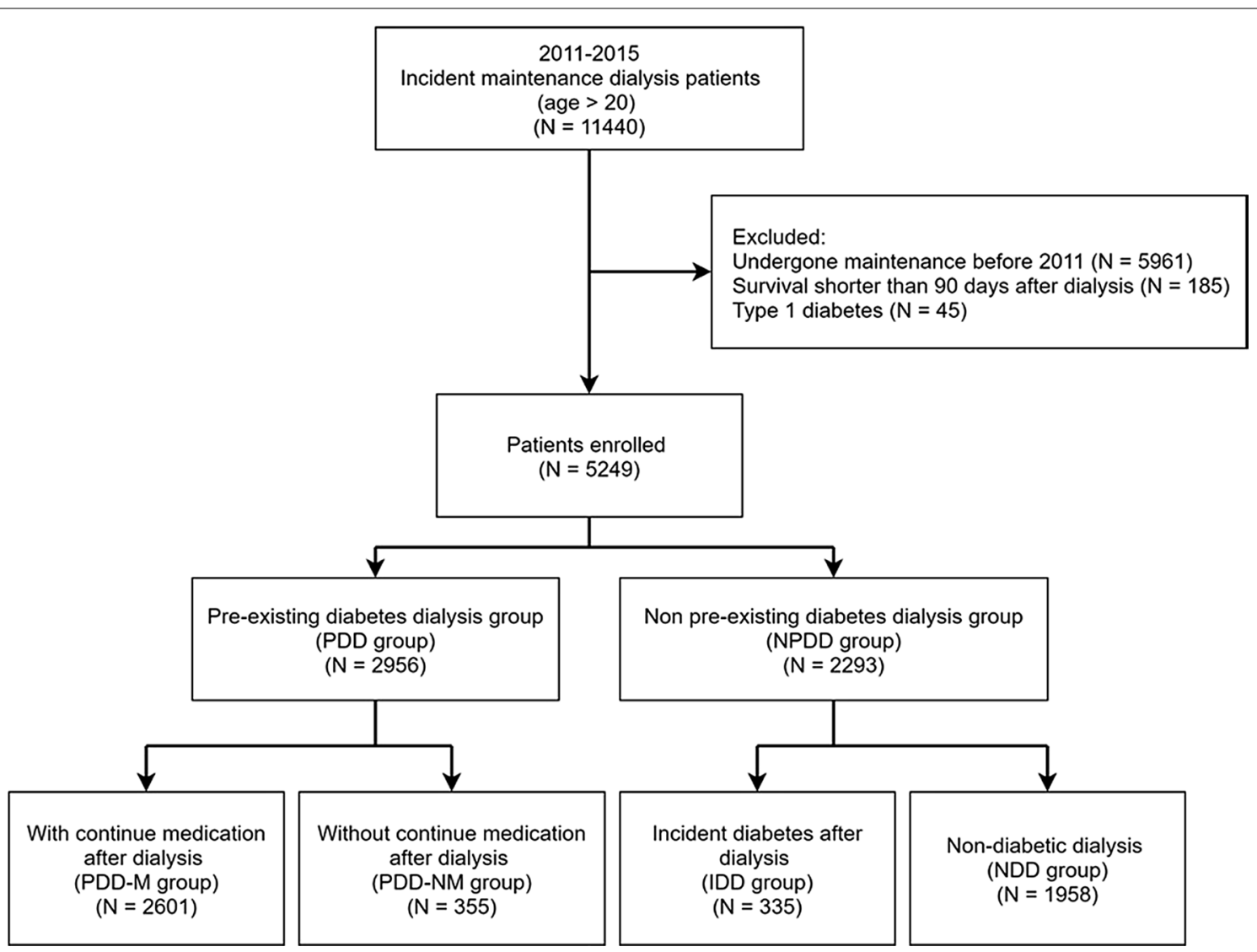

Fig. 1 Flow chart of enrolment of participants in the cohort study

12 months leading up to dialysis initiation $(\mathrm{P}<0.001)$, cerebrovascular accidents $(P=0.002)$, chronic liver disease $(P=0.001)$, and malignancy $(P=0.037)$. By contrast, a decreased risk of mortality was found in patients with hypertension $(\mathrm{P}=0.002)$ or those who were administered ACEI or ARB $(\mathrm{P}<0.001)$ or lipidlowering drugs $(\mathrm{P}<0.001)$.

Figure 3 shows that compared with the PDD-NM group, the PDD-M group had significantly lower risks of all-cause mortality, regardless of insulin (aHR: 0.66; CI $0.55-0.80 ; \mathrm{P}<0.001$ ) or OHA use (aHR: 0.56; CI $0.49-0.68 ; \mathrm{P}<0.001)$. Stratification analysis (Table 4 ) with different types of anti-diabetic drugs was applied. Results showed better survival benefit than non-users, regardless of the use of SU (aHR: 0.52; CI 0.41-0.67; $\mathrm{P}<0.001$ ), meglitinide (aHR: 0.48; CI 0.39-0.60; $\mathrm{P}<0.001$ ), TZD (aHR: 0.66; CI 0.65-1.25; $\mathrm{P}=0.02$ ), DPP-4 inhibitor (aHR: 0.40; CI 0.32-0.51; P $<0.001$ ), or insulin (aHR: 0.58; CI 0.47-0.68; P $<0.001$ ). Patients using DPP-4 inhibitors seemed to exhibit the greatest improvement.

\section{Analysis of patients with IDD during 2011-2015}

As shown in Table 5, factors that increased the risk of mortality in the IDD group included age older than 60 years $(\mathrm{P}<0.001)$, insulin use (compared with $\mathrm{OHA}$ use; $\mathrm{P}=0.008)$, hospitalization more than once during the 12 months leading up to dialysis initiation $(\mathrm{P}=0.031)$, cerebrovascular accidents $(\mathrm{P}=0.003)$, and chronic obstructive pulmonary disease $(\mathrm{P}=0.009)$. After stepwise multivariate regression analysis, significant independent predictors for increased mortality in the IDD group were age older than 60 years $(\mathrm{P}<0.001)$, cerebrovascular accidents $(P=0.003)$, and chronic obstructive pulmonary disease $(P=0.039)$. Patients with hyperlipidemia had a lower risk $(\mathrm{P}=0.014)$. Medication with insulin or OHA had no statistically significant difference in mortality in the overall IDD group. However, further stratification analysis (Table 6) indicated that OHA users had a better survival rate than insulin users in the older (aHR: 1.74; CI 1.09-2.77; $\mathrm{P}=0.02$ ) and male subgroups (aHR: 1.76; CI 0.99-3.11; $\mathrm{P}=0.05$ ). 
Table 1 Clinical characteristics and survival rates of dialysis patients during $2011-2015, n=5249$

\begin{tabular}{|c|c|c|c|c|c|}
\hline \multirow[t]{3}{*}{ Variable } & \multicolumn{2}{|l|}{ PDD } & \multicolumn{2}{|l|}{ NPDD } & \multirow[t]{3}{*}{ P-value } \\
\hline & PDD-M & PDD-NM & IDD & NDD & \\
\hline & $n=2601(49.6 \%)$ & $\mathrm{n}=355(6.8 \%)$ & $\mathrm{n}=335(6.4 \%)$ & $n=1958(37.3 \%)$ & \\
\hline Age, years (mean $\pm S D$ ) & $61 \pm 12$ & $64 \pm 13$ & $63 \pm 14$ & $61 \pm 16$ & 0.002 \\
\hline Gender & & & & & 0.561 \\
\hline Male & 1447 (56\%) & $200(56 \%)$ & $179(53 \%)$ & $1054(54 \%)$ & \\
\hline Female & $1154(44 \%)$ & $155(44 \%)$ & $156(47 \%)$ & $904(46 \%)$ & \\
\hline Region & & & & & 0.058 \\
\hline Northern & $1139(44 \%)$ & $146(41 \%)$ & 165 (49\%) & $850(43 \%)$ & \\
\hline Central & $617(24 \%)$ & $71(20 \%)$ & 77 (23\%) & 477 (24\%) & \\
\hline Southern/Eastern & $845(33 \%)$ & $138(39 \%)$ & $93(28 \%)$ & $631(32 \%)$ & \\
\hline Insured premium (NT\$) & & & & & 0.505 \\
\hline$<15,840$ & $635(24 \%)$ & $96(27 \%)$ & $96(29 \%)$ & 475 (24\%) & \\
\hline $15,840-25,000$ & $1187(46 \%)$ & $150(42 \%)$ & $145(43 \%)$ & $874(45 \%)$ & \\
\hline$>25,000$ & $779(30 \%)$ & $109(31 \%)$ & $94(28 \%)$ & $609(31 \%)$ & \\
\hline Urbanization & & & & & 0.775 \\
\hline Urban & $1460(56 \%)$ & $199(56 \%)$ & $179(53 \%)$ & $1116(57 \%)$ & \\
\hline Sub-urban & $835(32 \%)$ & $108(30 \%)$ & $117(35 \%)$ & $622(32 \%)$ & \\
\hline Rural & $306(12 \%)$ & $48(14 \%)$ & $39(12 \%)$ & $220(11 \%)$ & \\
\hline Hospital characteristics (teaching level) & & & & & $<0.001$ \\
\hline Medical center & $726(28 \%)$ & $91(26 \%)$ & $93(28 \%)$ & $668(34 \%)$ & \\
\hline Region & $886(34 \%)$ & $119(33 \%)$ & $113(34 \%)$ & $614(31 \%)$ & \\
\hline Others & $989(38 \%)$ & $145(41 \%)$ & $129(38 \%)$ & $676(35 \%)$ & \\
\hline $\begin{array}{l}\text { Number of admissions during the } \\
12 \text { months leading up to dialysis }\end{array}$ & & & & & $<0.001$ \\
\hline $0-1$ & $1744(67 \%)$ & $212(60 \%)$ & $274(82 \%)$ & $1574(80 \%)$ & \\
\hline$>1$ & $857(33 \%)$ & $143(40 \%)$ & $61(18 \%)$ & $384(20 \%)$ & \\
\hline Dialysis modality & & & & & $<0.001$ \\
\hline Hemodialysis & 2485 (95\%) & $340(96 \%)$ & $316(94 \%)$ & 1708 (87\%) & \\
\hline Peritoneal dialysis & $116(5 \%)$ & $15(4 \%)$ & $19(6 \%)$ & $250(13 \%)$ & \\
\hline \multicolumn{6}{|l|}{ Comorbidity } \\
\hline Hypertension & 1785 (69\%) & $270(76 \%)$ & $146(43 \%)$ & $987(50 \%)$ & $<0.001$ \\
\hline Hyperlipidemia & $790(30 \%)$ & $79(22 \%)$ & $58(17 \%)$ & $325(17 \%)$ & $<0.001$ \\
\hline Gout & $233(9 \%)$ & $50(14 \%)$ & $46(14 \%)$ & $338(17 \%)$ & $<0.001$ \\
\hline Congestive heart failure & $908(35 \%)$ & $133(38 \%)$ & $83(25 \%)$ & $501(26 \%)$ & $<0.001$ \\
\hline Cerebrovascular accident & $471(18 \%)$ & $57(16 \%)$ & $41(12 \%)$ & $208(11 \%)$ & $<0.001$ \\
\hline COPD & $185(7 \%)$ & $36(10 \%)$ & $34(10 \%)$ & $166(9 \%)$ & 0.053 \\
\hline Chronic liver disease & $149(6 \%)$ & $33(9 \%)$ & $19(6 \%)$ & $70(4 \%)$ & $<0.001$ \\
\hline Malignancy & $199(8 \%)$ & $45(13 \%)$ & $32(10 \%)$ & $212(11 \%)$ & $<0.001$ \\
\hline ACEI/ARB use & $1555(60 \%)$ & $97(27 \%)$ & $195(58 \%)$ & $878(45 \%)$ & $<0.001$ \\
\hline Lipid-lowering drug use & 1025 (39\%) & $45(13 \%)$ & $115(34 \%)$ & $431(22 \%)$ & $<0.001$ \\
\hline Mortality & $814(31 \%)$ & $166(47 \%)$ & $121(36 \%)$ & 495 (25\%) & $<0.001$ \\
\hline Survival, years (mean \pm SD) & $4.1 \pm 1.5$ & $3.0 \pm 2.1$ & $4.0 \pm 1.5$ & $4.2 \pm 1.6$ & $<0.001$ \\
\hline
\end{tabular}

$P D D$ pre-existing diabetes dialysis, NPDD non-pre-existing diabetes dialysis, $P D D-M$ pre-existing diabetes dialysis patients continuing medication, $P D D-N M$ preexisting diabetes dialysis patients not continuing medication, IDD incident diabetes after dialysis, NDD non-diabetic dialysis, COPD chronic obstructive pulmonary disease, $A C E l$ angiotensin converting enzyme inhibitors, $A R B$ angiotensin II receptor blockers

\section{Discussion}

The purpose of this retrospective cohort study using an $\mathrm{NHI}$ database was to investigate whether concomitant diabetes or anti-diabetic drug use affects the prognosis of dialysis patients. This epidemiological study provides a preliminary understanding of the importance of 
Table 2 Stepwise regression analysis for all dialysis patients with 5-year survival rate, $n=5249$

\begin{tabular}{|c|c|c|c|c|}
\hline \multirow[t]{2}{*}{ Variables } & \multicolumn{2}{|l|}{ Univariate } & \multicolumn{2}{|l|}{ Multivariate } \\
\hline & $\mathrm{HR}(95 \% \mathrm{Cl})$ & P-value & $\operatorname{aHR}(95 \% \mathrm{Cl})$ & P-value \\
\hline Age $>60$ year & $2.74(2.45-3.07)$ & $<0.001$ & $2.32(2.08-2.61)$ & $<0.001$ \\
\hline Gender-male & $1.06(0.96-1.18)$ & 0.273 & $1.00(0.95-1.05)$ & 0.984 \\
\hline \multicolumn{5}{|l|}{ Grouping } \\
\hline NDD & 1 & & 1 & \\
\hline $\mathrm{IDD}$ & $1.23(1.09-1.37)$ & $<0.001$ & $1.35(1.20-1.52)$ & $<0.001$ \\
\hline PDD-M & $1.46(1.19-1.78)$ & $<0.001$ & $1.52(1.25-1.86)$ & $<0.001$ \\
\hline PDD-NM & $2.58(2.16-3.07)$ & $<0.001$ & $2.12(1.77-2.53)$ & $<0.001$ \\
\hline \multicolumn{5}{|l|}{ Region } \\
\hline Northern & 1 & & & \\
\hline Central & $1.08(0.95-1.22)$ & 0.231 & & \\
\hline Southern/Eastern & $0.92(0.82-1.03)$ & 0.153 & & \\
\hline \multicolumn{5}{|l|}{ Insured premium (NT\$) } \\
\hline$<15,840$ & 1 & & 1 & \\
\hline $15,840-25,000$ & $0.84(0.74-0.94)$ & 0.003 & $0.86(0.75-0.96)$ & 0.010 \\
\hline$>25,000$ & $0.72(0.63-0.82)$ & $<0.001$ & $0.85(0.74-0.97)$ & 0.014 \\
\hline \multicolumn{5}{|l|}{ Urbanization } \\
\hline Urban & 1 & & & \\
\hline Sub-urban & $1.11(0.99-1.24)$ & 0.067 & & \\
\hline Rural & $1.23(1.05-1.43)$ & 0.008 & & \\
\hline \multicolumn{5}{|l|}{ Hospital characteristics } \\
\hline Medical center & 1 & & & \\
\hline Region/others & $1.01(0.91-1.13)$ & 0.836 & & \\
\hline \multicolumn{5}{|c|}{ Number of admissions during the 12 months leading up to dialysis } \\
\hline $0-1$ & 1 & & 1 & \\
\hline$>1$ & $1.87(1.69-2.07)$ & $<0.001$ & $1.60(1.42-1.80)$ & $<0.001$ \\
\hline \multicolumn{5}{|l|}{ Dialysis modality } \\
\hline Peritoneal dialysis & 1 & & & \\
\hline Hemodialysis & $1.76(1.40-2.22)$ & $<0.001$ & & \\
\hline \multicolumn{5}{|l|}{ Comorbidity } \\
\hline Hypertension & $1.22(1.10-1.36)$ & $<0.001$ & $0.78(0.69-0.88)$ & $<0.001$ \\
\hline Hyperlipidemia & $0.78(0.69-0.88)$ & $<0.001$ & & \\
\hline Gout & $0.91(0.78-1.07)$ & 0.251 & & \\
\hline Congestive heart failure & $1.37(1.23-1.52)$ & $<0.001$ & $1.15(1.03-1.28)$ & 0.012 \\
\hline Cerebrovascular accident & $1.51(1.33-1.71)$ & $<0.001$ & $1.26(1.11-1.43)$ & 0.001 \\
\hline COPD & $1.84(1.58-2.14)$ & $<0.001$ & $1.34(1.14-1.56)$ & $<0.001$ \\
\hline Chronic liver disease & $1.57(1.30-1.90)$ & $<0.001$ & $1.36(1.12-1.66)$ & 0.002 \\
\hline Malignancy & $1.81(1.57-2.09)$ & $<0.001$ & $1.39(1.20-1.61)$ & $<0.001$ \\
\hline ACEl/ARB use & $0.58(0.52-0.64)$ & $<0.001$ & $0.68(0.62-0.76)$ & $<0.001$ \\
\hline Lipid-lowering drug use & $0.50(0.44-0.57)$ & $<0.001$ & $0.61(0.54-0.69)$ & $<0.001$ \\
\hline
\end{tabular}

$H R$ hazard ratio, $C l$ confidence interval, $a H R$ adjusted hazard ratio, NDD non-diabetic dialysis, IDD incident diabetes after dialysis, $P D D$-NM pre-existing diabetes dialysis patients not continuing medication, $P D D-M$ pre-existing diabetes dialysis patients continuing medication, $C O P D$ chronic obstructive pulmonary disease, $A C E I$ angiotensin converting enzyme inhibitors, $A R B$ angiotensin II receptor blockers

diabetes disease control and drug selection in this group. Here, three issues were raised for discussion. First, dialysis patients with diabetes had higher mortality than those without, regardless of pre-existing diabetes at initiation of dialysis or new-onset diabetes after entering dialysis.
Second, for the PDD group, those who continued to use anti-diabetic medications after entering dialysis had a lower risk of death. In the subgroup analysis, patients using SU, meglitinide, TZD, DPP-4 inhibitor, or insulin all showed significantly better outcomes compared with 


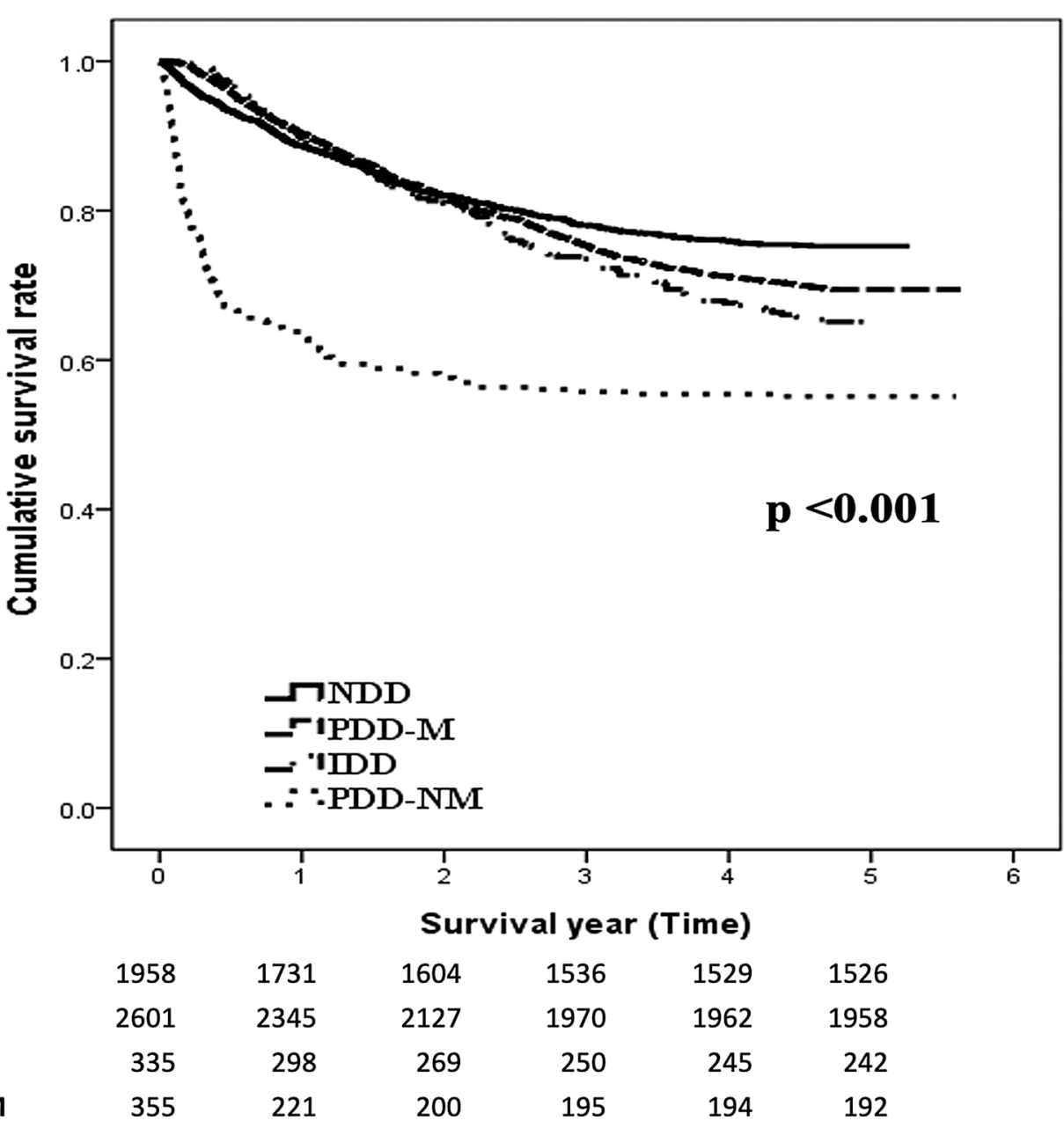

Fig. 2 The Kaplan-Meier survival curves between the four groups (PDD-M, PDD-NM, IDD, NDD)

non-users. Third, the choice of either insulin or OHA had no significant effect on overall survival in the new-onset diabetes after dialysis group. However, initial OHA use was associated with a better prognosis in the male and older than 60 years subgroups.

In our study, we confirmed that T2DM had a significant impact on the prognosis of patients undergoing dialysis. The previous study showed the survival of hemodialyzed diabetic patients was not inferior to nondiabetics [13]. On the contrary, our study result revealed that patients undergoing dialysis with pre-existing diabetes had a $32 \%$ higher mortality rate than NDD patients, and patients with new-onset diabetes after dialysis also had a $44 \%$ increased death risk. This finding was compatible with other study results in different countries [3, $14,15]$. Our results also confirmed that diabetes mellitus is independently associated with the risk of developing hypertension, cerebrovascular accidents, and congestive heart failure. Because diabetes has such a huge impact on the dialysis population, it should be actively treated and controlled.

One of the challenges regarding the management of diabetic dialysis patients is the uncertainty of blood sugar control goals. In the DOPPS study, the HbA1C level strongly predicted mortality in hemodialysis patients with type 1 or type 2 diabetes [16]. The association between $\mathrm{HbA} 1 \mathrm{C}$ and mortality was U-shaped, and the mortality was lowest at HbA1C 7-7.9\%. The KDOQI guideline also emphasized that in those dialysis patients with significant comorbidities or limited life expectancy, HbA1C control was preferably greater than 7, higher than in normal people [17]. However, another study also showed the effect of glycemic control in dialysis patients differed according to age and dialysis type and should be carefully planned and monitored [18]. Therefore, we suggested that glycemic control should be individualized assessment and management. Many important issues still need to be considered when we treat diabetic dialysis 
Table 3 Stepwise regression analysis for pre-existing diabetic dialysis patients with 5-year survival rate, $n=2956$

\begin{tabular}{|c|c|c|c|c|}
\hline \multirow[t]{2}{*}{ Variables } & \multicolumn{2}{|l|}{ Univariate } & \multicolumn{2}{|l|}{ Multivariate } \\
\hline & $\mathrm{HR}(95 \% \mathrm{Cl})$ & P-value & aHR $(95 \% \mathrm{Cl})$ & P-value \\
\hline Age $>60$ year & $2.31(2.01-2.65)$ & $<0.001$ & $2.05(1.78-2.35)$ & $<0.001$ \\
\hline Gender-male & $1.09(0.96-1.24)$ & 0.183 & & \\
\hline \multicolumn{5}{|l|}{ Anti-diabetic drug use } \\
\hline Non-user & 1 & & 1 & \\
\hline $\mathrm{OHA}$ & $0.44(0.37-0.53)$ & $<0.001$ & $0.56(0.49-0.68)$ & $<0.001$ \\
\hline Insulin & $0.52(0.43-0.63)$ & $<0.001$ & $0.66(0.55-0.80)$ & $<0.001$ \\
\hline \multicolumn{5}{|l|}{ Region } \\
\hline Northern & 1 & & & \\
\hline Central & $1.01(0.86-1.19)$ & 0.900 & & \\
\hline Southern/Eastern & $0.94(0.81-1.09)$ & 0.398 & & \\
\hline \multicolumn{5}{|l|}{ Insured premium (NT\$) } \\
\hline$<15,840$ & 1 & & & \\
\hline $15,840-25,000$ & $0.87(0.75-1.02)$ & 0.084 & & \\
\hline$>25,000$ & $0.80(0.68-0.95)$ & 0.012 & & \\
\hline \multicolumn{5}{|l|}{ Urbanization } \\
\hline Urban & 1 & & & \\
\hline Sub-urban & $1.09(0.95-1.25)$ & 0.236 & & \\
\hline Rural & $1.10(0.90-1.33)$ & 0.365 & & \\
\hline \multicolumn{5}{|l|}{ Hospital characteristics } \\
\hline Medical center & $1.03(0.89-1.19)$ & 0.674 & & \\
\hline Region/others & 1 & & & \\
\hline \multicolumn{5}{|c|}{ Number of admissions during the 12 months leading up to dialysis } \\
\hline $0-1$ & 1 & & 1 & \\
\hline$>1$ & $1.72(1.51-1.95)$ & $<0.001$ & $1.59(1.38-1.83)$ & $<0.001$ \\
\hline \multicolumn{5}{|l|}{ Dialysis modality } \\
\hline Peritoneal dialysis & 1 & & & \\
\hline Hemodialysis & $0.86(0.64-1.15)$ & 0.306 & & \\
\hline \multicolumn{5}{|l|}{ Comorbidity } \\
\hline Hypertension & $1.14(0.98-1.31)$ & 0.070 & $0.73(0.67-0.91)$ & 0.002 \\
\hline Hyperlipidemia & $0.80(0.69-0.93)$ & 0.003 & & \\
\hline Gout & $1.07(0.86-1.32)$ & 0.562 & & \\
\hline Congestive heart failure & $1.22(1.07-1.39)$ & 0.003 & & \\
\hline Cerebrovascular accident & $1.35(1.16-1.57)$ & $<0.001$ & $1.21(1.03-1.41)$ & 0.020 \\
\hline COPD & $1.57(1.27-1.64)$ & $<0.001$ & $1.21(0.98-1.49)$ & 0.087 \\
\hline Chronic liver disease & $1.65(1.32-2.07)$ & $<0.001$ & $1.48(1.18-1.86)$ & 0.001 \\
\hline Malignancy & $1.65(1.36-2.01)$ & $<0.001$ & $1.24(1.01-1.52)$ & 0.037 \\
\hline ACEI/ARB use & $0.59(0.52-0.67)$ & $<0.001$ & $0.56(0.47-0.68)$ & $<0.001$ \\
\hline Lipid-lowering drug use & $0.50(0.43-0.58)$ & $<0.001$ & $0.66(0.55-0.80)$ & $<0.001$ \\
\hline
\end{tabular}

$H R$ hazard ratio, $C l$ confidence interval, aHR adjusted hazard ratio, $O H A$ oral hypoglycemia agents, COPD chronic obstructive pulmonary disease, $A C E I$ angiotensin converting enzyme inhibitors, $A R B$ angiotensin II receptor blockers

patients, including the accuracy of HbA1C measurement in the renal failure population $[19,20]$, the burn-out diabetes phenomenon [21], and whether strict blood sugar control would be too late to improve cardiovascular benefits [22].

Few diabetic patients may experience spontaneous resolution of hyperglycemia status and fluctuating HbA1C levels to as low as $<6 \%$ after entering dialysis. Some experts $[9,23]$ defined this phenomenon as "burn-out diabetes". Multiple factors may contribute to this condition, including malnutrition, protein-energy wasting, prolongation of insulin half-life due to decreased clearance, reduction of renal gluconeogenesis, accumulation of uremic toxin, and glucose removal by hemodialysis 


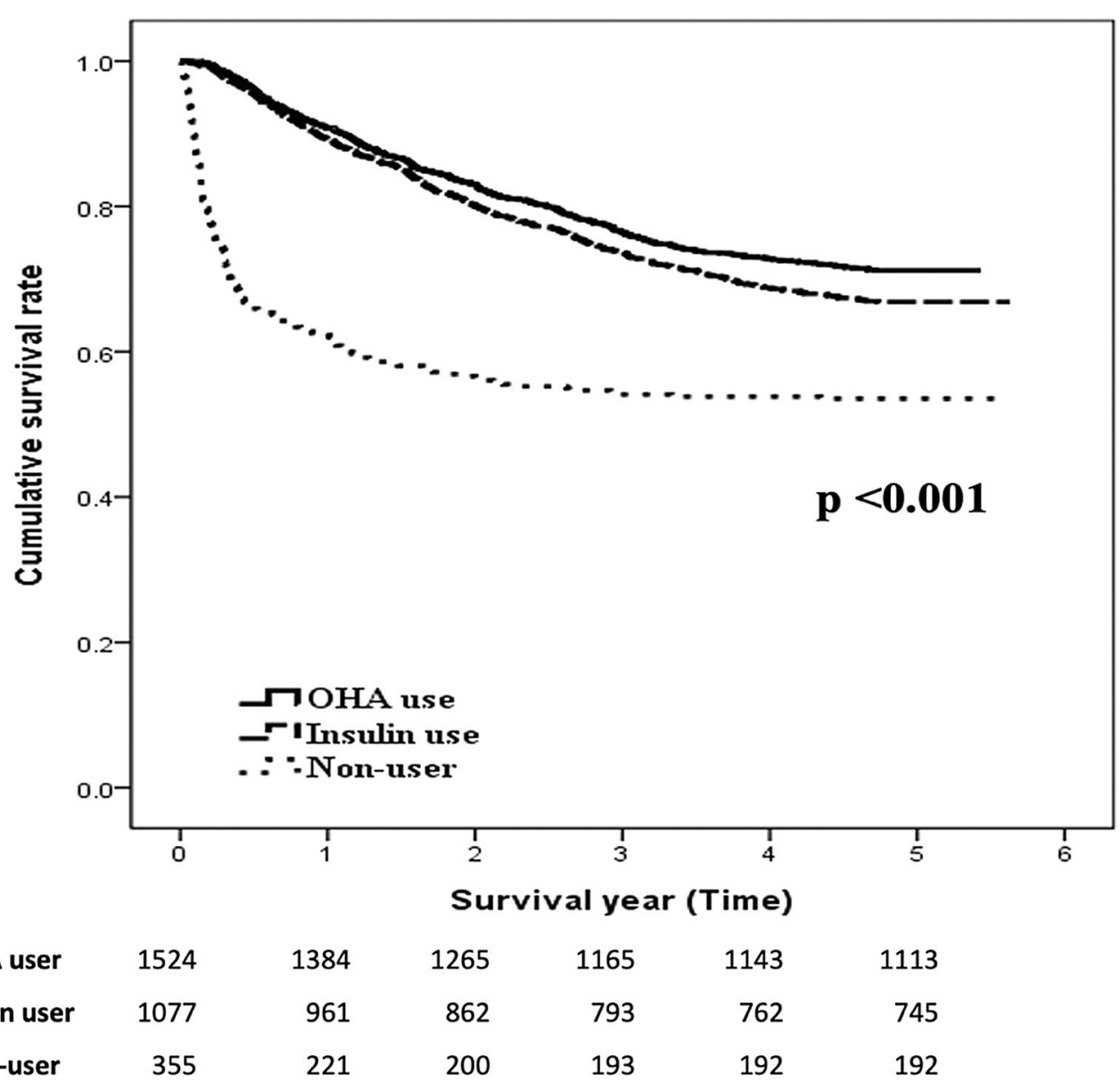

Fig. 3 The Kaplan-Meier survival curves for the anti-diabetic drug use subgroups in PDD patients

Table 4 Stratification analysis for the survival rate of pre-existing diabetic dialysis patients with different types of anti-diabetic drugs. $n=2956$

\begin{tabular}{llr}
\hline Variables & aHR $(95 \% \mathrm{Cl})$ & P-value \\
\hline Anti-diabetic drugs & & \\
Non-user & 1 & $<0.001$ \\
SU & $0.52(0.41-0.67)$ & 0.020 \\
TZD & $0.66(0.65-1.25)$ & $<0.001$ \\
Meglitinide & $0.48(0.39-0.60)$ & $<0.001$ \\
DPP-4 inhibitors & $0.40(0.32-0.51)$ & $<0.001$ \\
Insulin & $0.58(0.47-0.68)$ &
\end{tabular}

aHR adjusted hazard ratio, $\mathrm{Cl}$ confidence interval, SU sulfonylurea, TZD thiazolidinedione, DPP-4 dipeptidyl-peptidase IV

[7]. Anti-diabetic medications like insulin or other OHAs were suggested to be discontinued to minimize the risk of hypoglycemia in this situation [24]. Although etiological analysis is lacking, we believe the burn-out phenomenon is one of the most important reasons for withdrawal of anti-diabetic medication after initiation of dialysis. However, the adverse effects of insulin resistance in such patients are continuing progression despite near-normal HbA1C levels. In our study, patients who discontinued medication were older, had a higher prevalence of congestive heart failure, malignancy, and chronic liver disease, and there was less RAS blockade usage, all of which have been described as obvious determinants of mortality in dialyzed individuals. However, after these factors and the number of hospitalizations in the past year were adjusted for, continued anti-diabetic drug use was still an independent predictor of favorable survival rate. Thus, we speculate that anti-diabetic medication use has possible additional benefits to directly improve outcomes, such as decreased insulin resistance, improved endothelial function, and anti-inflammatory effects $[25,26]$. The actual mechanisms of this association still need more research to explore.

Our subgroup analysis also showed that patients using SU, meglitinide, TZD, DPP-4 inhibitor, or insulin all had significantly better outcomes compared with non-users. 
Table 5 Stepwise regression analysis for incident diabetic patients after dialysis with 5-year survival rate, $\mathrm{n}=335$

\begin{tabular}{|c|c|c|c|c|}
\hline \multirow[t]{2}{*}{ Variables } & \multicolumn{2}{|l|}{ Univariate } & \multicolumn{2}{|l|}{ Multivariate } \\
\hline & $\mathrm{HR}(95 \% \mathrm{Cl})$ & P-value & aHR $(95 \% \mathrm{Cl})$ & P-value \\
\hline Age $>60$ years & $2.52(1.67-3.79)$ & $<0.001$ & $2.35(1.56-3.55)$ & $<0.001$ \\
\hline Gender-male & $1.40(0.98-2.00)$ & 0.068 & & \\
\hline \multicolumn{5}{|l|}{ Anti-diabetic drug use } \\
\hline $\mathrm{OHA}$ & 1 & & & \\
\hline Insulin & $1.63(1.14-2.33)$ & 0.008 & $1.41(0.98-2.02)$ & 0.068 \\
\hline \multicolumn{5}{|l|}{ Region } \\
\hline Northern & 1 & & & \\
\hline Central & $1.23(0.80-1.89)$ & 0.900 & & \\
\hline Southern/Eastern & $0.94(0.63-1.52)$ & 0.398 & & \\
\hline \multicolumn{5}{|l|}{ Insured premium (NT\$) } \\
\hline$<15,840$ & 1 & & & \\
\hline $15,840-25,000$ & $0.78(0.51-1.20)$ & 0.254 & & \\
\hline$>25,000$ & $0.87(0.55-1.38)$ & 0.543 & & \\
\hline \multicolumn{5}{|l|}{ Urbanization } \\
\hline Urban & 1 & & & \\
\hline Sub-urban & $0.75(0.50-1.12)$ & 0.157 & & \\
\hline Rural & $0.84(0.47-1.62)$ & 0.565 & & \\
\hline \multicolumn{5}{|l|}{ Hospital characteristics } \\
\hline Medical center & $1.19(0.81-1.76)$ & 0.378 & & \\
\hline Region/others & 1 & & & \\
\hline \multicolumn{5}{|c|}{ Number of admissions during the 12 months leading up to dialysis } \\
\hline $0-1$ & 1 & & & \\
\hline$>1$ & $1.59(1.05-2.43)$ & 0.031 & & \\
\hline \multicolumn{5}{|l|}{ Dialysis modality } \\
\hline Peritoneal dialysis & 1 & & & \\
\hline Hemodialysis & $1.24(0.54-2.81)$ & 0.614 & & \\
\hline \multicolumn{5}{|l|}{ Comorbidity } \\
\hline Hypertension & $1.22(0.85-1.75)$ & 0.275 & & \\
\hline Hyperlipidemia & $0.43(0.23-0.81)$ & 0.008 & $0.45(0.24-0.85)$ & 0.014 \\
\hline Gout & $0.85(0.50-1.46)$ & 0.557 & & \\
\hline Congestive heart failure & $1.27(0.85-1.89)$ & 0.239 & & \\
\hline Cerebrovascular accident & $2.03(1.28-3.22)$ & 0.003 & $2.04(1.27-3.29)$ & 0.003 \\
\hline COPD & $1.92(1.18-3.14)$ & 0.009 & $1.68(1.03-2.76)$ & 0.039 \\
\hline Chronic liver disease & $1.00(0.47-2.15)$ & 0.998 & & \\
\hline Malignancy & $1.65(0.98-2.80)$ & 0.061 & & \\
\hline ACEI/ARB use & $0.68(0.48-0.97)$ & 0.033 & & \\
\hline Lipid-lowering drugs use & $0.50(0.33-0.76)$ & 0.001 & $0.66(0.43-1.02)$ & 0.060 \\
\hline
\end{tabular}

$H R$ hazard ratio, $\mathrm{Cl}$ confidence interval, aHR adjusted hazard ratio, $O H A$ oral hypoglycemia agents, COPD chronic obstructive pulmonary disease, $A C E I$ angiotensin converting enzyme inhibitors, $A R B$ angiotensin II receptor blockers

Balancing anti-diabetic medication administration and hypoglycemic risk is an important issue. In this situation, the use of DPP-4 inhibitors appears to be more reasonable and valuable. Compatible with our finding, Chan et al. indicated that DPP-4 inhibitor users in diabetic dialysis patients had a lower risk of all-cause mortality than nonusers [27]. The survival benefits may be attributable to significantly fewer ischemic stroke events among DPP-4 inhibitor users due to neuroprotective effects. DPP-4 inhibitors have a greater reduction in HbA1C than in fasting blood sugar, which can lower the possible side effect of hypoglycemia and reduce blood glucose variability $[28,29]$. In addition, DPP-4 inhibitors resulted in fewer weight gain side effects, more protective of pancreatic beta-cell function and volume, and better tolerability [30]. An additional benefit for dialysis patients includes 
Table 6 Stratification analysis for incident diabetic patients after dialysis with 5-year survival rate, $n=335$

\begin{tabular}{llc}
\hline Variables & aHR $(\mathbf{9 5} \% \mathbf{C l})$ & P-value \\
\hline Age & & \\
$>60$ years & 1 & \\
OHA & $1.74(1.09-2.77)$ & 0.020 \\
Insulin & & \\
$\leq 60$ years & 1 & 0.855 \\
OHA & $1.08(0.46-2.57)$ & \\
Insulin & & \\
Sex & 1 & 0.050 \\
Male & $1.76(0.99-3.11)$ & \\
OHA & 1 & 0.758 \\
Insulin & $1.09(0.62-1.95)$ & \\
Female & & \\
OHA & & \\
Insulin &
\end{tabular}

aHR adjusted hazard ratio, $\mathrm{OHA}$ oral hypoglycemia agents

reduced inter-dialytic weight accumulation [31]. We suggest that DPP-4 inhibitors are an appropriate and suitable choice for diabetic dialysis patients who are at high risk of hypoglycemia, regarding monotherapy or add-on treatment.

According to a previous report [32], the prevalence of new-onset diabetes after dialysis was around $12.7 \%$ within a ten-year follow-up period; the incidence was significantly associated with female sex, age, and baseline comorbidity. The 5-year incidence in our study was $14.6 \%$. We further analyzed the relationship between different anti-diabetic medication use in patients with new-onset diabetes after dialysis and mortality, with the results indicating that initial treatment with insulin or other OHAs did not affect the outcome. Stratification analysis revealed that OHA users had a better survival rate than insulin users only in the old and male subgroups. These groups are traditionally susceptible to cardiovascular disease and cerebrovascular disease [33]. Some studies showed OHA has a better protection effect than insulin use [34, 35]. Another possible explanation is that patients who started on insulin injection treatment usually had poor blood sugar control, implying an increased susceptibility to large vessel complications [36]. Our results are worthy of further larger randomized trials to verify their significance.

There are some limitations to our study. First, this observational cohort study using the NHIRD could only show associations and not explain direct causality. However, epidemiological studies can provide multivariate comparisons and reveal specific impact factors, then there can be further prospective studies or randomized controlled trials to confirm causality. Second, because all definitions of variables were based on clinical physicians' diagnosis and coding, misidentification may result in bias. Third, the NHIRD does not provide personal history, lifestyle information, and laboratory results-such as HbA1C or serial glucose level-which are potential confounding factors for this study, and this is a major inherent limitation in the study. Fourth, we did not explore the relationship between the reasons for drug withdrawal and the cause of death in our study. Last, we grouped drug type based on the first drug used more than 90-days and we did not evaluate long-term medication compliance. This problem requires a more precise randomized clinical trial to build more solid evidence.

\section{Conclusions}

Our study used an NHI database to conduct large-scale epidemiological research on the incident maintenance dialysis group. We found that dialysis patients with diabetes certainly had a higher mortality rate, regardless of whether diabetes was pre-existing or new-onset. Our novel finding was that continued use of anti-diabetic drugs after dialysis and choice of medication may affect clinical outcomes. Further rigorous studies are needed to confirm the trends and associations discovered by our research.

\section{Abbreviations \\ ACEl: Angiotensin-converting enzyme inhibitors; ARB: Angiotensin II recep- tor blockers; DPP-4: Dipeptidyl-peptidase IV; ESRD: End-stage renal disease; HbA1C: Hemoglobin A1C; HWDC: Health and Welfare Data Science Center; IDD: Incident diabetes after dialysis.; KDOQI: Kidney Disease Outcomes Quality Initiative; LHID2010: Longitudinal Health Insurance Database 2010; NDD: Non-diabetes dialysis; NPDD: Non-pre-existing diabetes dialysis group; NHI: National Health Insurance; NHIRD: National Health Insurance Research Database; OHA: Oral hypoglycemic agents; PDD: Pre-existing diabetes dialysis group; PDD-M: Pre-existing diabetes dialysis patients continuing medication; PDD-NM: Pre-existing diabetes dialysis patients not continuing medication; SU: Sulfonylureas; T2DM: Type 2 diabetes mellitus; TZD: Thiazolidinediones.}

\section{Supplementary Information}

The online version contains supplementary material available at https://doi. org/10.1186/s12933-021-01364-w.

Additional file 1: Table S1. ICD-9 and ICD-10 codes used to identify comorbidities. Table S2. Anatomic therapeutic chemical (ATC) classification codes for select medications.

\section{Acknowledgements}

The authors would like to thank the staff at the Cancer Center, Department of Medical Education and Research, and Research Center of Medical Informatics of Kaohsiung Veterans General Hospital for providing information in response to inquiries and assistance in the data processing.

\section{Authors' contributions}

SHO, CHY, and JSC: study concept and design, analysis and interpretation of data, acquisition of data and drafting of the manuscript, and study 
supervision; HYC, NWF, and CLC: analysis and interpretation of data. All authors read and approved the final manuscript.

\section{Funding}

This work was supported by grants from Kaohsiung Veterans General Hospital (KSVGH 110-001 to S. H. Ou and KSVGH 110-136 to J. S. Chen).

\section{Availability of data and materials}

The data used to support the findings of this study are available from the corresponding author upon reasonable request.

\section{Declarations}

\section{Ethics approval and consent to participate}

This study was also approved by the Institutional Review Board of the Kaohsiung Veterans General Hospital (IRB No.: KSVGH18-CT10-07), who supervised the study in accordance with the tenets of the Declaration of Helsinki (1975) and its later amendment (2013). The requirement for patient consent was waived due to retrospective study design and proved by Institutional Review Board of the Kaohsiung Veterans General Hospital.

\section{Consent for publication}

\section{Not applicable.}

\section{Competing interests}

The authors declare no conflict of interest.

\section{Author details}

${ }^{1}$ Division of Nephrology, Department of Internal Medicine, Kaohsiung Veterans General Hospital, Kaohsiung, Taiwan. ${ }^{2}$ Faculty of Medicine, School of Medicine, National Yang Ming Chiao Tung University, Taipei, Taiwan. ${ }^{3}$ Graduate Institute of Clinical Medicine, Kaohsiung Medical University, Kaohsiung, Taiwan. ${ }^{4}$ Department of Pediatrics, Kaohsiung Veterans General Hospital, Kaohsiung, Taiwan. ${ }^{5}$ Department of Medical Education and Research, Kaohsiung Veterans General Hospital, Kaohsiung, Taiwan. ${ }^{6}$ Faculty of Medicine, School of Medicine, National Defense Medicine, Taipei, Taiwan.

Received: 27 May 2021 Accepted: 15 August 2021

Published online: 08 September 2021

\section{References}

1. Hoffmann F, Haastert B, Koch M, Giani G, Glaeske G, Icks A. The effect of diabetes on incidence and mortality in end-stage renal disease in Germany. Nephrol Dial Transplant. 2011;26:1634-40.

2. Schena FP. Epidemiology of end-stage renal disease: international comparisons of renal replacement therapy. Kidney Int. 2000;57:39-45.

3. Soleymanian T, Kokabeh Z, Ramaghi R, Mahjoub A, Argani H. Clinical outcomes and quality of life in hemodialysis diabetic patients versus non-diabetics. J Nephropathol. 2016;6:81-9.

4. Zhan X, Wen Y, Zhou Q, Feng X, Peng FF, Wang N, et al. Co-existence of diabetes mellitus and pre-existing cardiovascular disease, diabetes mellitus, and pre-existing cardiovascular disease and mortality in peritoneal dialysis patients. Res Sq. 2020. https://doi.org/10.21203/rs.3. rs-35743/v1.

5. Holman RR, Paul SK, Bethel MA, et al. 10-year follow-up of intensive glucose control in type 2 diabetes. N Engl J Med. 2008;359:1577-89.

6. Oomichi T, Emoto M, Tabata T, Morioka T, Tsujimoto Y, Tahara H, et al. Impact of glycemic control on survival of diabetic patients on chronic regular hemodialysis: a 7-year observational study. Diabetes Care. 2006;29:1496-500.

7. Rhee CM, Leung AM, Kovesdy CP, Lynch KE, Brent GA, Kalantar-Zadeh K. Updates on the management of diabetes in dialysis patients. Semin Dial. 2014;27:135-45.

8. Gianchandani RY, Neupane S, lyengar JJ, Heung M. Pathophysiology and management of hypoglycemia in end-stage renal disease patients: a review. Endocr Pract. 2017:23:353-62.
9. Park J, Lertdumrongluk P, Molnar MZ, Kovesdy CP, Kalantar-Zadeh K. Glycemic control in diabetic dialysis patients and the burnt-out diabetes phenomenon. Curr Diab Rep. 2012;12:432-9.

10. Inker LA, Astor BC, Fox CH, Isakova T, Lash JP, Peralta CA, et al. KDOQI US commentary on the $2012 \mathrm{KDIGO}$ clinical practice guideline for the evaluation and management of CKD. Am J Kidney Dis. 2014;63:713-35.

11. Lin CC, Lai MS, Syu CY, Chang SC, Tseng FY. Accuracy of diabetes diagnosis in health insurance claims data in Taiwan. J Formos Med Assoc. 2005;104:157-63.

12. Shih C-J, Chen Y-T, Ou S-M, Yang W-C, Kuo S-C, Tarng D-C, et al. The impact of dialysis therapy on older patients with advanced chronic kidney disease: a nationwide population-based study. BMC Med. 2014:12:169.

13. Tomaszuk-Kazberuk A, Bachorzewska-Gajewska H, Malyszko J, Malyszko J, Mysliwiec M, Musial WJ. Impact of diabetes mellitus on survival in patients with end-stage renal disease: a three-year follow-up. Kidney Blood Press Res. 2011;34:83-6.

14. Schroijen MA, Dekkers OM, Grootendorst DC, Noordzij M, Romijn JA, Krediet RT, et al. Survival in dialysis patients is not different between patients with diabetes as primary renal disease and patients with diabetes as a co-morbid condition. BMC Nephrol. 2011;12:1-7.

15. Rački S, Zaputović L, Vujicić B, Crnčević-Orlić Ž, Dvornik Š, Mavrić Ž. Comparison of survival between diabetic and non-diabetic patients on maintenance hemodialysis: a single-center experience. Diabetes Res Clin Pract. 2007;75:169-75.

16. Ramirez SP, McCullough KP, Thumma JR, Nelson RG, Morgenstern $\mathrm{H}$, Gillespie BW", et al. Hemoglobin A1c levels and mortality in the diabetic hemodialysis population: findings from the dialysis outcomes and practice patterns study (DOPPS). Diabetes Care. 2012;35:2527-32.

17. Guideline Development Group, Bilo H, Coentrão L, Couchoud C, Covic A, De Sutter J, Drechsler C, et al. Clinical practice guideline on management of patients with diabetes and chronic kidney disease stage $3 \mathrm{~b}$ or higher (eGFR < 45 mL/min). Nephrol Dial Transplant. 2015;30:ii1-142.

18. Park Jl, Bae E, Kim YL, Kang SW, Yang CW, Kim NH, et al. Glycemic control and mortality in diabetic patients undergoing dialysis focusing on the effects of age and dialysis type: a prospective cohort study in Korea. PloS ONE. 2015;10:e0136085.

19. Little RR, Rohlfing CL, Tennill AL, Hanson SE, Connolly S, Higgins T, et al. Measurement of $\mathrm{HbA} 1 \mathrm{c}$ in patients with chronic renal failure. Clin Chim Acta. 2013;418:73-6.

20. Speeckaert M, Van Biesen W, Delanghe J, Slingerland R, Wiecek A, Heaf $J$, et al. Are there better alternatives than haemoglobin A1c to estimate glycaemic control in the chronic kidney disease population? Nephrol Dial Transplant. 2014;29:2167-77.

21. Dozio E, Corradi V, Proglio M, Vianello E, Menicanti L, Rigolini R, et al. Usefulness of glycated albumin as a biomarker for glucose control and prognostic factor in chronic kidney disease patients on dialysis (CKDG5D). Diabetes Res Clin Pract. 2018;140:9-17.

22. Galindo RJ, Beck RW, Scioscia MF, Umpierrez GE, Tuttle KR. Glycemic monitoring and management in advanced chronic kidney disease. Endocr Rev. 2020:41:756-74.

23. Kovesdy CP, Park JC, Kalantar-Zadeh K. Glycemic control and burnt-out diabetes in ESRD. Semin Dial. 2010;23:148-56.

24. Anjani A, Devi NH, Nagaraj RD, Siva KV. Burnt-out diabetes in diabetic nephropathy patients on maintenance haemodialysis. J Clin Sci Res. 2019;8:7-10.

25. Pereira CA, Carneiro FS, Matsumoto T, Tostes RC. Bonus effects of antidiabetic drugs: possible beneficial effects on endothelial dysfunction, vascular inflammation and atherosclerosis. Basic Clin Pharmacol Toxicol. 2018;123:523-38.

26. Scheen A, Esser N, Paquot N. Antidiabetic agents: potential antiinflammatory activity beyond glucose control. Diabetes Metab. 2015;41:183-94.

27. Chan S-Y, Ou S-M, Chen Y-T, Shih C-J. Effects of DPP-4 inhibitors on cardiovascular outcomes in patients with type 2 diabetes and end-stage renal disease. Int J Cardiol. 2016;218:170-5.

28. Abe M, Okada K. DPP-4 inhibitors in diabetic patients with chronic kidney disease and end-stage kidney disease on dialysis in clinical practice. In: Nitta K, editor. Chronic kidney diseases-recent advances in clinical and basic research. Switzerland: Karger Publishers; 2015. p. 98-115. 
29. Farngren J, Ahrén B. Incretin-based medications (GLP-1 receptor agonists, DPP-4 inhibitors) as a means to avoid hypoglycaemic episodes. Metabolism. 2019;99:25-31.

30. Liu D, Jin B, Chen W, Yun P. Dipeptidyl peptidase 4 (DPP-4) inhibitors and cardiovascular outcomes in patients with type 2 diabetes mellitus (T2DM): a systematic review and meta-analysis. BMC Pharmacol Toxicol. 2019;20:15.

31. Fujii Y, Abe M, Higuchi T, Mizuno M, Suzuki H, Matsumoto S, et al. The dipeptidyl peptidase-4 inhibitor alogliptin improves glycemic control in type 2 diabetic patients undergoing hemodialysis. Expert Opin Pharmacother. 2013;14:259-67.

32. Tien KJ, Lin ZZ, Chio CC, Wang JJ, Chu CC, Sun YM, et al. Epidemiology and mortality of new-onset diabetes after dialysis: Taiwan national cohort study. Diabetes Care. 2013;36:3027-32.

33. Rodgers JL, Jones J, Bolleddu SI, Vanthenapalli S, Rodgers LE, Shah K, et al. Cardiovascular risks associated with gender and aging. J Cardiovasc Dev Dis. 2019;6:19
34. Wang MT, Lin SC, Tang PL, Hung WT, Cheng CC, Yang JS, et al. The impact of DPP-4 inhibitors on long-term survival among diabetic patients after first acute myocardial infarction. Cardiovasc Diabetol. 2017;16:1-11.

35. Hsiao P-J, Wu K-L, Chiu S-H, Chan J-S, Lin Y-F, Wu C-Z, et al. Impact of the use of anti-diabetic drugs on survival of diabetic dialysis patients: a 5-year retrospective cohort study in Taiwan. Clin Exp Nephrol. 2017;21:694-704.

36. Agrawal RP, Ola V, Bishnoi P, Gothwal S, Sirohi P, Agrawal R. Prevalence of micro and macrovascular complications and their risk factors in type-2 diabetes mellitus. J Assoc Physicians India. 2014;62:504-8.

\section{Publisher's Note}

Springer Nature remains neutral with regard to jurisdictional claims in published maps and institutional affiliations.
Ready to submit your research? Choose BMC and benefit from:

- fast, convenient online submission

- thorough peer review by experienced researchers in your field

- rapid publication on acceptance

- support for research data, including large and complex data types

- gold Open Access which fosters wider collaboration and increased citations

- maximum visibility for your research: over $100 \mathrm{M}$ website views per year

At BMC, research is always in progress.

Learn more biomedcentral.com/submissions 